\title{
Editorial
}

\section{Retos y desafíos de las universidades 2020-2030}

\author{
Escrito por: \\ Diego Felipe Arbeláez-Campillo \\ https://orcid.org/0000-0002-9041-9563 \\ Grupo de investigación Lenguajes, representaciones y Educación, \\ Universidad de la Amazonia, Colombia. \\ Magda Julissa Rojas-Bahamón \\ https://orcid.org/0000-0003-4882-1476 \\ $\mathrm{PhD}$. Educación y cultura ambiental. Docente IE Jorge Eliecer Gaitán, \\ Universidad de la Amazonia, Colombia.
}

La comunidad académica y las instituciones de educación superior (universidades) afrontan varios desafíos en los próximos decenios. Algunos de estos grandes desafíos es el reconocer, adaptar e integrarse con la inteligencia artificial (IA) en la enseñanza, el aprendizaje y el ámbito administrativo. Así es, las universidades deben reconocer que la IA ya se encuentra presente en nuestras vidas y que su avance se desarrolla a un ritmo exponencial. También deben ser conscientes de que es necesaria su adaptabilidad por parte de estudiantes, profesores y administrativos y su integración en los procesos de aprendizaje, enseñanza y administración en las universidades.

Sin embargo, aunque la adaptabilidad de la IE en los diversos ámbitos es necesaria, es importante que la sociedad y la comunidad científica realicen procesos de evaluación y crítica a los sesgos algorítmicos presentes en la inteligencia artificial. De acuerdo a estudios realizados, se ha podido evidenciar, que a los igual que los humanos, los algoritmos involucran prejuicios raciales, sexistas, xenofóbicos, homofóbicos... perpetuando de alguna manera, la afectación a los grupos históricamente desfavorecidos como las mujeres, las personas negras y de otras razas diferentes a las blancas.

En ese orden de ideas, Bolukbasi et al. (2016) sostiene que se aplicaron técnicas de aprendizaje automático para entrenar a una inteligencia artificial utilizando Google News. Como producto de este ejercicio, se resolvió la analogía "hombre es a programador de ordenadores lo que mujeres a $x$ ". La respuesta automática fue que " $x$ = ama de casa". Así mismo, el estudio de (Sheng et. al, 2019) se evidencio que si se daba como inicio la frase "el hombre blanco trabaja como...", la IA la completaba con "un oficial de policía”. En cambio, si el comienzo de la frase era "el hombre negro trabaja como...", el algoritmo generaba el texto "un proxeneta durante 15 días". Otras investigaciones han podido evidenciar los errores que se encuentran presentes en las nuevas tecnologías, motivo por el cual es imperante que en los procesos educativos haya intervención humana y no sean las máquinas las que trasladen a los profesores en sus actividades de enseñanza, aunque los estados y las grandes empresas tecnológicas propendan porque los profesores sean remplazados por inteligencia artificial en menos de diez años.

Es por esta razón que se han adelantado estudios como la Dubai Future Academy en la que se abordó ¿Cómo será la educación y el aprendizaje entre 2020 y 2050 ? Desde esta perspectiva, Vidal (2019) sostiene que en 2022 la atmósfera de la Tierra o la de la Luna se verán cómo se puede ver un paisaje o una selva en la realidad, los niños en sus clases usaran gafas de realidad virtual y realmente verán aquello que estudian como si fuera un objeto real. De la misma forma, manifiesta que en 2025 la realidad virtual y la realidad aumentada aumentarán el aprendizaje remoto y, como resultado, las aulas comenzarán a desaparecer y para 2030 los cerebros de los humanos se conectarán a la nube (transhumanos). La capacidad de respaldar nuestros pensamientos y recuerdos utilizando computadoras aumentará dramáticamente nuestro potencial de aprendizaje. En 2030 también la imagen cerebral revoluciona nuestra enseñanza. El uso de imágenes cerebrales nos permitirá afinar la educación al probar que modos de enseñanza funcionan mejor con cada alumno. En 2031 la educación ya sólo será personalizada, empezará una personalización del estudio totalmente mejorada, los estudiantes pasarán mucho tiempo involucrando a los profesores individualmente y 
se ejecutarán como tutorías individuales de un modo totalmente virtual pero tremendamente real en cuanto a la percepción sensorial. Ese mismo 2031 nuestros maestros serán en gran medida pura Inteligencia Artificial, de hecho, será inteligencia cognitiva.

El científico informático Eric Cooke asegura que, en los próximos 15 años, las máquinas inteligentes reemplazarán en gran medida a los maestros humanos, por eficiencia, capacidad y efectividad. En 2035 los sistemas de aprendizaje artificiales nos brindarán capacidades de comprensión específicas y temporales: es decir, podrán permitir que una persona comprenda temporalmente o hable un idioma extranjero con fluidez. En 2036, es decir en apenas dos décadas veremos cómo desaparecen casi todas las instituciones educativas tal y como ahora las conocemos. Las mejores universidades seguirán, aunque en un escenario muy diferente. Las de nivel medio apenas existirán. Para 2036 los expertos predicen que se abandonarán los métodos tradicionales de prueba $\mathrm{y}$ comenzaremos a centrarnos en evaluaciones de otro tipo, sin notas, sin análisis de resultados. La idea tendrá más que ver con el nivel de comprensión a partir del uso tecnológico. En 2043 la educación será una parte dominante de nuestras vidas. Nuestro empleo no será un trabajo, tendrá mucho más parecido a ir a la escuela a aprender constantemente. Tendremos acceso constante a toda la información del mundo a través de nuestros dispositivos confundidos en nuestro cuerpo, por lo que la educación se volverá más omnipresente a medida que sigamos evolucionando. (Vidal, 2019)

Ante este panorama, las universidades requieren incentivar el pensamiento crítico en los estudiantes. Así mismo, en los currículos se requiere el plantear problemas del entorno local y las soluciones a estos problemas. Lo anterior, busca crear en los estudiantes la innovación y la creatividad para transformar y replantear lo existente. Las universidades tienen un gran desafío y para esto se requiere articularse con todos los sectores económicos, productivos y de servicios.

Por otro lado, es necesario realizar alianzas estratégicas entre las diferentes empresas nacionales e internacionales con las universidades. Lo anterior, con la búsqueda de un objetivo común que es la solución a los problemas de la sociedad. Es por esto que tanto los empresarios, sociedad requieren un dialogo y un trabajo en equipo con las universidades. Dado que los requerimientos de solución a problemas y necesidades que presenten las empresas y las sociedades sean solucionados por las universidades en los salones de clase, laboratorios y la misma empresa. Lo anterior, ayuda a estimular la investigación y es una fuente de recursos para las universidades.

\section{Los retos de la educación y de la sociedad que deben afrontar los profesores.}

Los grandes cambios que demanda la sociedad, tienen en la educación un gran desafío y son los docentes universitarios los llamados a repensar sus formas de enseñanza. De acuerdo a lo anterior, Espejo y Marty (2020) manifiestan debemos cuestionarnos a nosotros mismos, los maestros. ¿Cómo me estoy preparando para estos retos? ¿Qué paradigmas debo romper para adaptarme a un mundo que me exige lo mismo que necesito desarrollar en mis alumnos? ¿Cuáles están siendo las fuentes de información que me permiten mejorar mi práctica docente? ¿Con quién y de que tipo realizo alianzas para llevar mejores experiencias de aprendizaje a mis alumnos? Lo anterior, nos hace pensar que se requieren profesores con amplio conocimiento es su área del saber, pero también requiere que tenga conocimiento en saberes didácticos y pedagógicos que permita que su saber disciplinar sea entendido y compartido con sus estudiantes. Así mismo, se requiere de profesores con formación en investigación y solución de problemas, profesores universitarios con publicación de artículos científicos en bases de datos reconocidas como Wos, que participen en ponencias y en grupos de investigación. Profesores con la capacidad de conocer aceptar los últimos avances tecnológicos, de innovar, de replantear y proponer un mejor mundo para los seres de este planeta.

\section{Capacidad para afrontar el reto GAFAM (Google, Amazon, Facebook, Apple y Microsoft).}

Al consultar en la internet sobre este acrónimo GAFAM se encuentra una palabra que llama la atención: "conquista", una palabra que denota lo que buscan estas empresas, luchar y ganar un campo en el sector de la educación. Es decir, estas cinco transnacionales tecnológicas son una gran competencia y desafío para las universidades, dada su abundante oferta de cursos de poca duración, gratuitos o de bajo costo e innovadores; estos cursos certifican al estudiante por pocos años o revalidan las acreditaciones de los estudiantes. Por tal motivo, se requiere que las universidades visualicen este competidor y posible conquistador de los 


\section{AMAZONDA}

territorios de la educación. De acuerdo a lo anterior, las estrategias utilizadas por las GAFAM, para colonizar la educación, según SantanillaLab (2021), son:

Conectan vida real y vida escolar, es por eso preguntarse ¿Conectan la vida real y escolar las universidades?

Conocen al usuario, ¿Conocen al usuario de sus servicios las universidades?

Ofrecen una garantía, puesto que la formación está respaldada por marcas de reconocido prestigio con presencia sólida en distintos sectores e industrias. ¿Por qué no van a ofrecer un producto y un servicio excelente en el mundo educativo? Además, acompañan sus implantaciones escolares de licencias y servicios de formación gratuitos y acompañamiento online $\mathrm{y}$ offline muy potentes.

Han establecido nuevas rutinas de uso

¿Google Drive puede ser un portafolio de evidencias de aprendizaje para un grupo que está trabajando por proyectos? La respuesta es sí. Estas herramientas están tan refinadas desde el punto de vista de experiencia de uso y están tan presentes en distintos contextos de nuestras vidas, que han construido prácticas que el mundo educativo está adoptando.

Han cambiado hábitos: La llegada de nuevas herramientas conlleva la aparición de nuevas formas de trabajo, nuevos modos de comportamiento... y ¿nuevas formas de pensar?

Tienen un aura innovadora, y lo han hecho bajo un doble paradigma. Desde un punto de vista profesional docente, gusta cada vez más participar en un programa de certificación de Google o Apple porque se asocia con innovación y vanguardia.

Venden una falsa gratuidad. En muchas ocasiones las GAFAM se acercan a los centros educativos ofreciendo sus servicios de forma gratuita. Meras estrategias comerciales y/o la punta de lanza de sus verdaderos propósitos: conocer a sus usuarios también en contextos escolares, apropiarse de la solución a las necesidades de la comunidad educativa y generar negocio.

Te atrapan en su ecosistema: Hardware, software, herramientas, formación, certificación, etc. Cuando caes en su tela de araña sueles quedar atrapado. $\mathrm{Y}$ con menos posibilidades de conectarte a otros ecosistemas de lo que aparentemente pueda parecer.

También te atrapan en su comunidad de usuarios. $\mathrm{O}$ en lo que aparentemente parece una comunidad de personas que, como docente, genera una sensación de pertenencia y de confianza. Pero... ¿de verdad hay comunidades consolidadas?

Es por esta razón que las universidades deben responderse si algunas de estas estrategias que utilizan las GAFAM, las piensan implementar o ya las implementado.

\section{Otro de los grandes retos de las universidades: la internacionalización.}

Considerando lo planteado en el apartado anterior, urge una universidad pensada en un mundo globalizado y sin fronteras. Universidades que realicen convenios internacionales con otras universidades con la figura de consorcio (Universidades consorciadas) que permitan la movilidad entre carreras y universidades y que como resultado se pueda obtener la doble titulación de los estudiantes, la disminución de tiempos de estudio, la continuidad de posgrados tanto en maestrías como doctorados, Del mismo modo, estos convenios permiten la movilidad de estudiantes y docentes cada uno en su rol y redundara en el conocimiento de la diversidad de culturas, de necesidades de las sociedad y de pensar de forma global.

\section{Creación de nuevas carreras}

Gran parte de las profesiones universitarias están pensadas para resolver problemas a una sociedad industrial y no a una sociedad tecnológica. Es por este motivo, que se requiere la creación de nuevas carreras que permitan resolver los nuevos retos que ya se están presentando tales como: la ciberseguridad, big data, predictores del mercado, experto en minería de datos, expertos en nuevos alimentos, ingenieros civiles $\mathrm{y}$ arquitectos en materiales y urbanismo en sociedades tecnológicas entre otros.

\section{Referencias}

Bolukbasi, T., Chang, K.W., Zou, J., Saligrama, V., \& Kala, A. (2016). Man is to Computer Programmer as Woman is to Homemaker? Debiasing Word Embeddings. arXiv preprint arXiv. https://arxiv.org/pdf/1607.06520.pdf 
Espejo, M.E \& Marty, P. (2020) Objetivos de desarrollo sostenible, ¿un reto para la educación? Tecnologico de Monterrey. https://observatorio.tec.mx/edu-bitsblog/objetivos-de-desarrollo-sostenible-retoeducacion-agenda2030

Santillanalab (2021). GAFAM, el inquietante interés de las grandes tecnológicas por la educación. https://www.santillanalab.com/gafam-interestecnologicas-educacion/

Sheng, E., Chang, K.W., Natarajan, P., \& Peng, N. (2019). The Woman Worked as a Babysitter: On Biases in Language Generation. arXiv preprint arXiv

Vidal, M. (2019). La educación del futuro. Desde 2020 al 2050. Marcvidal. https://www.marcvidal.net/blog/2019/5/29/laeducacion-futuro-2020-2050 